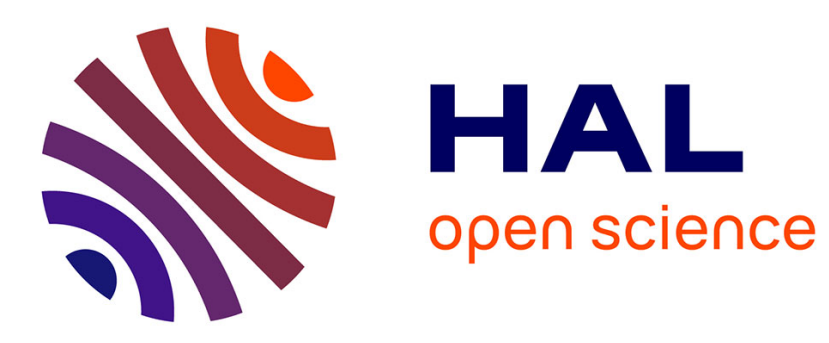

\title{
Towards Probing a Variation of Fundamental Constants with Optical Clock Transitions of 127I2
}

\author{
Florin Lucian Constantin
}

\section{To cite this version:}

Florin Lucian Constantin. Towards Probing a Variation of Fundamental Constants with Optical Clock Transitions of 127I2. 2020 Joint Conference of the IEEE International Frequency Control Symposium and International Symposium on Applications of Ferroelectrics (IFCS-ISAF), Jul 2020, Keystone, United States. pp.1-3, 10.1109/IFCS-ISAF41089.2020.9234878 . hal-03055497

\section{HAL Id: hal-03055497 https://hal.science/hal-03055497}

Submitted on 11 Dec 2020

HAL is a multi-disciplinary open access archive for the deposit and dissemination of scientific research documents, whether they are published or not. The documents may come from teaching and research institutions in France or abroad, or from public or private research centers.
L'archive ouverte pluridisciplinaire HAL, est destinée au dépôt et à la diffusion de documents scientifiques de niveau recherche, publiés ou non, émanant des établissements d'enseignement et de recherche français ou étrangers, des laboratoires publics ou privés.

$$
\text { Copyright }
$$




\title{
Towards Probing a Variation of Fundamental Constants with Optical Clock Transitions of ${ }^{127} \mathrm{I}_{2}$
}

\author{
Florin Lucian Constantin \\ Laboratoire PhLAM, CNRS UMR 8523 \\ CNRS, University Lille 1 \\ Villeneuve d'Ascq, France \\ FL.Constantin@univ-lille1.fr
}

\begin{abstract}
Precision measurements of the molecular iodine optical transitions can be exploited for constraining a possible time variation of the fundamental constants. The sensitivities of the molecular frequencies to a variation of the proton-toelectron mass ratio and of the fine structure constant are calculated. Compact molecular iodine clocks, designed for space applications with improved stability performances, enable fractional frequency reproducibility at the $10^{-15}$ level. The comparison of the optical iodine clocks based on transitions at $532 \mathrm{~nm}, 514 \mathrm{~nm}$ and $502 \mathrm{~nm}$ with the Cs frequency standard can constrain a fractional time variation of the proton-to-electron mass ratio and of the fine structure constant at the $10^{-14} \mathrm{yr}^{-1}$ level.
\end{abstract}

Keywords-Laser Spectroscopy, Clocks, Rovibronic States, Fundamental Constants

\section{INTRODUCTION}

A space-time variability of fundamental constants, allowed in theories beyond the Standard Model and General Relativity, can infirm the validity of the local position invariance hypothesis and the equivalence principle [1]. In unified theories, the variation of a constant is associated to the variation of other constants. A possible time variation of fundamental constants was tested in laboratory using comparisons between different atomic clocks. The most precise results allowed to constrain a fractional time variation of the fine structure constant $\alpha$ at the $10^{-17} \mathrm{yr}^{-1}$ level and of the proton-to-electron mass ratio $\mu$ at the $10^{-16} \mathrm{yr}^{-1}$ level $[2,3]$. The optical atomic clocks are not sensitive to the variation of $\mu$. In this case one should address the case of the molecular transitions which are intrinsically sensitive to the $\mu$ variation. The absolute frequency measurements of a rovibrational transition of $\mathrm{SF}_{6}$ allowed to determine a modelfree constraint of the fractional time variation of $\mu$ at $5.6 \times$ $10^{-14} \mathrm{yr}^{-1}$ [4]. The strategy for improving the constraint with laboratory tests is to increase the accuracy of the clock transitions, to exploit transitions that are highly sensitive to the variations of fundamental constants, and to perform measurements during a long period of time.

This contribution addresses the sensitivity to variations of the fundamental constants from the molecular iodine optical transitions spanning the spectral domain between $500 \mathrm{~nm}$ and $700 \mathrm{~nm}$. The Doppler-free lines of molecular iodine form a dense grid of optical frequency references that were exploited to build frequency standards [5].

\section{Sensitivity to VARIATIONS OF $\alpha$ AND $\mu$ FROM THE OPTICAL TRANSITIONS OF THE MOLECULAR IODINE}

The rovibronic energy levels of ${ }^{127} \mathrm{I}_{2}$ in the ground $\mathrm{X}$ electronic state and in the excited $\mathrm{B}$ electronic state are described with the Dunham series expansion :

$$
\begin{aligned}
& E_{X}\left(v^{\prime \prime}, J^{\prime \prime}\right)=\sum_{k, l} Y_{k, l}^{X}\left(v^{\prime \prime}+\frac{1}{2}\right)^{k}\left[J^{\prime \prime}\left(J^{\prime \prime}+1\right)\right]^{l} \\
& E_{B}\left(v^{\prime}, J^{\prime}\right)=T_{e}+\sum_{k, l} Y_{k, l}^{B}\left(v^{\prime}+\frac{1}{2}\right)^{k}\left[J^{\prime}\left(J^{\prime}+1\right)\right]^{l}
\end{aligned}
$$

in function of the vibrational quantum numbers $v^{\prime}$ and $v^{\prime}$,' the rotational quantum numbers $J^{\prime}$ and $J^{\prime \prime}$, the electronic energy $T_{e}$, and the Dunham parameters $Y_{k, l}^{X, B}$. The sensitivity of the energy levels to a variation of $\mu$ arises solely from the dependence of the Dunham parameters on the nuclear mass :

$$
\begin{aligned}
& \frac{d \ln Y_{k, l}^{B, X}}{d \ln \mu}=-\left(\frac{k}{2}+l\right) \\
& \frac{d \ln T_{e}}{d \ln \mu}=0
\end{aligned}
$$

The rovibronic energy levels of the $\mathrm{X}$ and $\mathrm{B}$ electronic states of ${ }^{127} \mathrm{I}_{2}$ are issued from the fine structure splitting of the iodine atom, that depends quadratically on the atomic number and the fine structure constant.

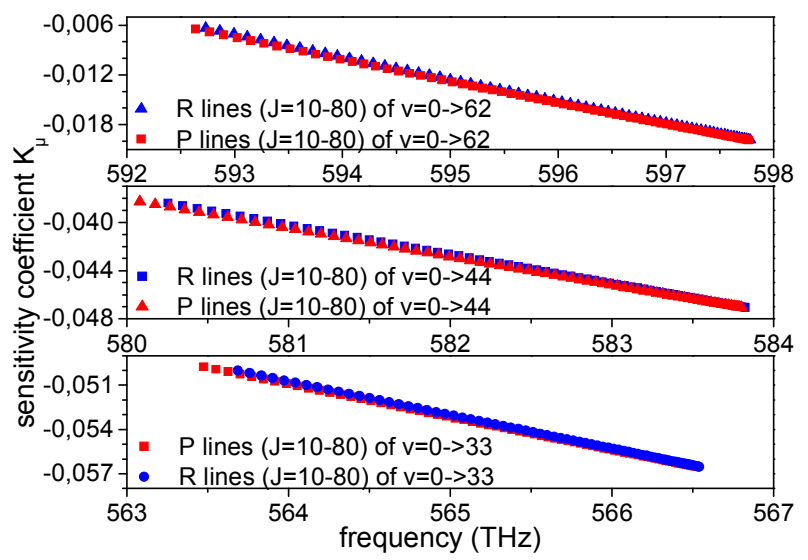

Fig. 1. Sensitivity coefficients to a variation of $\mu$ from selected optical transitions of ${ }^{127} \mathrm{I}_{2}$.

The parameters of interest in this contribution are the adimensional sensitivity coefficients of the ${ }^{127} \mathrm{I}_{2}$ transitions to a variation of the constants. The sensitivity coefficients to a variation of $\alpha$ are assumed here to be the same as for the case of the iodine atom $K_{\alpha}=2$. The sensitivity coefficients to a variation of $\mu$ are calculated using eq. $(1,2)$ and the molecular parameters from [6]. The results are plotted in Fig. 1 for a selection of molecular iodine transitions. 


\section{METROLOGICAL PERFORMANCES WITH COMPACT CLOCKS BASED ON THE MOLECULAR IODINE TRANSITIONS}

Compact optical setups with lasers stabilized on the molecular iodine transitions have been developed in view of several space applications $[7,8]$, including gravitational wave detection and test of the equivalence principle. The approaches are based on lasers with low intrinsic phase noise which are frequency doubled or tripled to be locked onto molecular iodine lines with high signal to noise ratio and high quality factor that are probed with the frequency modulation transfer technique. The transitions to the dissociation limit of the $\mathrm{B}$ electronic state have the advantage to display narrow natural linewidths [9]. A setup developed at Laboratoire SYRTE (Fig. 2) exploits a narrow linewidth Telecom laser source that is frequency tripled using the fibered waveguide periodically-poled lithium niobate (PPLN) technology and locked to a narrow line at $514 \mathrm{~nm}$ [7].

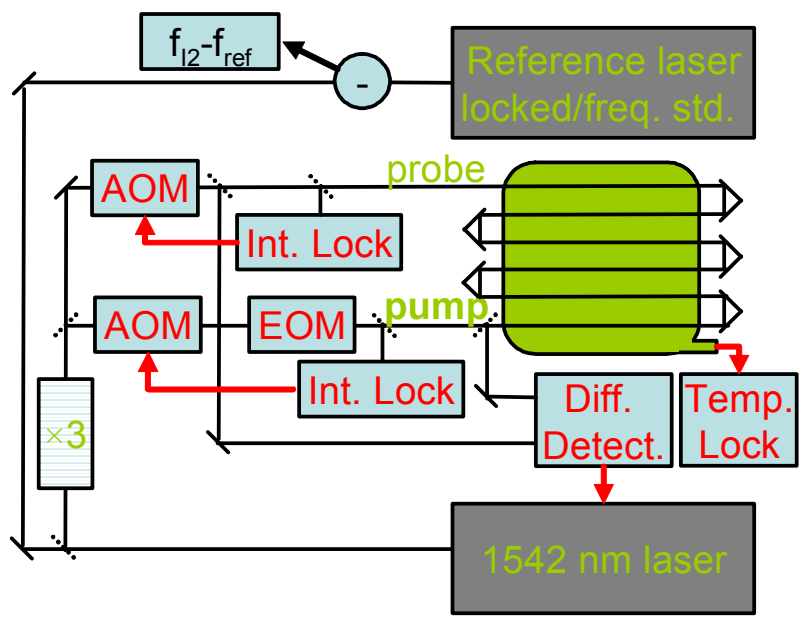

Fig. 2. An experimental setup for the molecular iodine clock. AOM acousto-optical modulator, EOM electro-optical modulator, x3 frequency tripling stage based on waveguide PPLN crystals.

The molecular iodine stabilized laser is compared with a similar Telecom laser locked onto an ultrastable optical cavity [10], for which the frequency is compared to the SYRTE's frequency standards. The stabilization of the pump and probe laser intensities, the strict control of the residual amplitude modulation and the stabilization of the cold finger temperature of the cell allowed to reach an Allan deviation of $2.9 \times 10^{-14} \tau^{-1 / 2}$ with a minimum of $5.7 \times 10^{-15}$ for $100 \mathrm{~s}$ of integration time [7]. The reproducibility of laser frequency was estimated fractionally at the $10^{-15}$ level [11]. Comparable metrological performances have been obtained with a semiconductor laser that was frequency doubled and locked to a molecular iodine line at $532 \mathrm{~nm}$ [8].

\section{ESTIMATED CONSTRAINTS FOR THE TIME VARIATIONS OF $\alpha$}

\section{AND $\mu$ FROM MOLECULAR IODINE CLOCK COMPARISONS}

The time variation of the ratio between the iodine molecular clock frequency and the Cs clock frequency may be expressed in function of the time variations of $\mu$ and $\alpha$ as :

$$
\frac{1}{f_{I_{2}} / f_{C s}} \frac{d\left(f_{I_{2}} / f_{C s}\right)}{d t}=\left(K_{\mu}^{I_{2}}+1\right) \frac{1}{\mu} \frac{d \mu}{d t}+\left(K_{\alpha}^{I_{2}}-K_{\alpha}^{C s}\right) \frac{1}{\alpha} \frac{d \alpha}{d t}(3)
$$

by introducing the sensitivity coefficients of the Cs clock to a variation of $\alpha, K_{\alpha}^{C s}=2.83$, and to a variation of $\mu$, $K_{\mu}^{C s}=-1$, respectively. The last equation neglects the contributions arising from the time variation of the relevant nuclear g-factors.

TABLE I. MOLECULAR IODINE TRANSITIONS SELECTED FOR ABSOLUTE FREQUENCY MEASUREMENTS

\begin{tabular}{|c|c|c|c|}
\hline${ }^{127} \mathbf{I}_{\mathbf{2}}$ clock transition & Exp. freq. (THz) & $\mathbf{K}_{\boldsymbol{\mu}}$ & $\mathbf{K}_{\boldsymbol{\alpha}}$ \\
\hline${ }^{127} \mathrm{I}_{2}(1): \mathrm{R}(26) 62-0$ & 597.366 & -0.019 & 2 \\
\hline${ }^{127} \mathrm{I}_{2}(2): \mathrm{R}(35) 44-0$ & 583.254 & -0.045 & 2 \\
\hline${ }^{127} \mathrm{I}_{2}(3): \mathrm{R}(56) 32-0$ & 563.260 & -0.054 & 2 \\
\hline
\end{tabular}

This contribution evaluates the constraints to the variations of $\alpha$ and $\mu$ that can be inferred from absolute frequency measurements of the three molecular iodine transitions that are indicated in Table 1 . The time variations of the absolute molecular iodine frequencies are described with three eq. (3) with the relevant sensitivity coefficients. The estimation is based on 100 frequency measurements performed with the same periodicity during one year. The fractional frequency uncertainty of the iodine frequency, assumed at $10^{-15}$, translates into a fractional uncertainty of the time derivative estimated at $4 \times 10^{-16} \mathrm{yr}^{-1}$. For this choice of clock comparisons, the uncertainties of the time derivatives of the fundamental constants are :

$$
\begin{aligned}
& u\left(\frac{1}{\mu} \frac{d \mu}{d t}\right)=1.6 \times 10^{-14} y r^{-1} \\
& u\left(\frac{1}{\alpha} \frac{d \alpha}{d t}\right)=1.8 \times 10^{-14} y r^{-1}
\end{aligned}
$$

The proposed approach will therefore enable a threefold improvement of the constraint on the variation of $\mu$ that has been determined by absolute frequency measurements of a rovibrational transition [4]. Moreover, the molecular iodine frequency measurements can allow constraining for the first time the time variation of $\alpha$ with a molecular clock, independently on previous optical atomic clock comparisons.

\section{CONCLUSION}

This contribution proposes a new method to probe the variability of fundamental constants with precision measurements in molecular physics. The optical clocks based on the molecular iodine transitions are sensitive to the variations of the proton-to-electron mass ratio and the fine structure constant and may allow $10^{-15}$ reproducibility. The absolute frequency measurements for a set of molecular iodine transitions may constrain conjointly the time variations of the proton-to-electron mass ratio and the fine structure constant at the $10^{-14} \mathrm{yr}^{-1}$ level.

\section{ACKNOWLEDGEMENT}

This work was supported by the LABEX Cluster of Excellence FIRST-TF (ANR-10-LABX-48-01) within the Program «Investissements d'Avenir» operated by the 
French National Research Agency. Laboratoire PhLAM of the University of Lille is UMR 8523 of the CNRS.

\section{REFERENCES}

[1] J.-P. Uzan, "Varying constants, gravitation and cosmology," Living Rev. Relativ., vol. 14, p. 2, March 2011.

[2] R.M. Godun et al, "Frequency ratio of two optical clock transitions in ${ }^{171} \mathrm{Yb}^{+}$and constraints on the time variation of fundamental constants," Phys. Rev. Lett., vol. 113, p. 210801, November 2014.

[3] N. Huntemann et al, "Improved limit on a temporal variation of $\mathrm{m}_{\mathrm{p}} / \mathrm{m}_{\mathrm{e}}$ from comparisons of $\mathrm{Yb}^{+}$and Cs atomic clocks," Phys. Rev. Lett., vol. 113, p. 210802, November 2014.

[4] A. Shelkovnikov, R. J. Butcher, C. Chardonnet, and A. Amy-Klein, "Stability of the proton-to-electron mass ratio," Phys. Rev. Lett., vol. 100, p. 150801, April 2008.

[5] F. Riehle, P. Gill, F. Arias, and L. Robertsson, "The CIPM list of recommended frequency standard values: guidelines and procedures," Metrologia, vol. 55, pp. 188-200, February 2018.
[6] S. Gerstenkom and P. Luc, "Description of the absorption spectrum of iodine recorded by means of Fourier transform spectroscopy : the (BX) system,” J. Phys. France, vol. 46, pp. 867-881, June 1985.

[7] J Barbarat et al, "Compact and transportable iodine frequency stabilized laser," Proc. of SPIE, International Conference on Space Optics - ICSO 2018, vol. 11180, p. 111800T, July 2019.

[8] N. Gürlebeck et al, "BOOST: A satellite mission to test Lorentz invariance using high-performance optical frequency references," Phys. Rev. D, vol. 97, p. 124051, June 2018.

[9] W.-Y. Cheng, L. Chen, T.H. Yoon, J.L. Hall, and J. Ye, "SubDoppler molecular-iodine transitions near the dissociation limit (523498 nm)," Opt. Lett., vol. 27, pp. 571-573, April 2002.

[10] B. Argence et al, "Prototype of an ultra-stable optical cavity for space applications," Opt. Express, vol. 20, p. 25409, November 2012.

[11] C. Philippe, "Sources laser à $1,5 \mu \mathrm{m}$ stabilisées en fréquence sur l'iode moléculaire," unpublished. 